\title{
RECURRENCE RELATIONS FOR QUOTIENT \\ MOMENTS OF GENERALIZED PARETO \\ DISTRIBUTION BASED ON GENERALIZED ORDER STATISTICS AND CHARACTERIZATION
}

\author{
DEVENDRA KUMAR*
}

\begin{abstract}
Generalized Pareto distribution play an important role in reliability, extreme value theory, and other branches of applied probability and statistics. This family of distribution includes exponential distribution, Pareto or Lomax distribution. In this paper, we established exact expressions and recurrence relations satisfied by the quotient moments of generalized order statistics for a generalized Pareto distribution. Further the results for quotient moments of order statistics and records are deduced from the relations obtained and a theorem for characterizing this distribution is presented.
\end{abstract}

\section{Introduction}

Kamps [7] introduced the concept of generalized order statistics (gos) as follows: Let $X_{1}, X_{2} \ldots$ be a sequence of independent and identically distributed (iid) random variables $(r v)$ with absolutely continuous distribution function $(d f) F(x)$ and probability density function $(p d f), f(x)$, $x \in(\alpha, \beta)$. Let $n \in N, n \geq 2, k>0, m \in \Re$, be the parameters such that

$$
\gamma_{r}=k+(n-r)(m+1)>0, \text { for all } r \in\{1,2, \ldots, n-1\},
$$

Received December 18, 2013; Accepted June 30, 2014.

2010 Mathematics Subject Classification: Primary 62G30, 62E10.

Key words and phrases: generalized order statistics, order statistics, record values, generalized Pareto distribution, recurrence relations, conditional expectation and characterization. 
where $M_{r}=\sum_{j=r}^{n-1} m j$. Then $X(1, n, m, k), \ldots, X(n, n, m, k), r=1,2, \ldots n$ are called gos if their joint $p d f$ is given by

$$
k\left(\prod_{j=1}^{n-1} \gamma_{j}\right)\left(\prod_{i=1}^{n-1}\left[1-F\left(x_{i}\right)\right]^{m} f\left(x_{i}\right)\right)\left[1-F\left(x_{n}\right)\right]^{k-1} f\left(x_{n}\right)
$$

on the cone $F^{-1}(0)<x_{1} \leq x_{2} \leq \ldots \leq x_{n}<F^{-1}(1)$.

The model of gos contains as special cases of order statistics, record values and sequential order statistics.

Choosing the parameters appropriately (Cramer, [4], we get the variant of the gos given in Table 1.

\begin{tabular}{|c|c|c|c|}
\hline & $\gamma_{\boldsymbol{n}}=\boldsymbol{k}$ & $\boldsymbol{\gamma}_{\boldsymbol{r}}$ & $\boldsymbol{m}_{\boldsymbol{r}}$ \\
\hline i) Sequential order statistics & $\alpha_{n}$ & $(n-r+1) \alpha_{r}$ & $\gamma_{r}-\gamma_{r+1}-1$ \\
\hline ii) Ordinary order statistics & 1 & $n-r+1$ & 0 \\
\hline ii) Record values & 1 & 1 & -1 \\
\hline $\begin{array}{c}\text { iv) Progressively type II } \\
\text { censored order statistics }\end{array}$ & $R_{n}+1$ & $n-r+1+\sum_{j=r}^{n} R_{j}$ & $R_{r}$ \\
\hline $\begin{array}{c}\text { v) Pfeifer's record values } \\
\text { vilut }\end{array}$ & $\beta_{n}$ & $\beta_{r}$ & $\beta_{r}-\beta_{r+1}-1$ \\
\hline
\end{tabular}

TABLE 1. Variants of the generalized order statistics

For simplicity we shall assume $m_{1}=m_{2}=\ldots=m_{n-1}=m$.

The $p d f$ of the $r$-th $g o s, X(r, n, m, k), 1 \leq r \leq n$, is

$$
f_{X(r, n, m, k)}(x)=\frac{C_{r-1}}{(r-1) !}[\bar{F}(x)]^{\gamma_{r}-1} f(x) g_{m}^{r-1}(F(x))
$$

and the joint $p d f$ of $X(r, n, m, k)$ and $X(s, n, m, k), 1 \leq r<s \leq n$, is

$$
\begin{aligned}
& f_{X(r, n, m, k), X(s, n, m, k)}(x, y) \\
& =\frac{C_{s-1}}{(r-1) !(s-r-1) !}[\bar{F}(x)]^{m} f(x) g_{m}^{r-1}(F(x)) \\
& \quad \times\left[h_{m}(F(y))-h_{m}(F(x))\right]^{s-r-1}[\bar{F}(y)]^{\gamma_{s}-1} f(y), x<y,
\end{aligned}
$$

where

$$
\bar{F}(x)=1-F(x), C_{r-1}=\prod_{i=1}^{r} \gamma_{i}, \gamma_{i}=k+(n-i)(m+1),
$$




$$
h_{m}(x)=\left\{\begin{array}{l}
-\frac{1}{m+1}(1-x)^{m+1}, m \neq-1 \\
-\ln (1-x), m=-1
\end{array}\right.
$$

and

$$
g_{m}(x)=h_{m}(x)-h_{m}(1), x \in[0,1) .
$$

Let $X(r, n, m, k), r=1,2, \ldots, n$ be $g o s$ from a continuous population with $d f F(x)$ and $p d f f(x)$. Then the conditional $p d f$ of $X(s, n, m, k)$ given $X(r, n, m, k)=x, 1 \leq r<s \leq n$, in view of (1.2) and (1.3), is

$$
\begin{aligned}
& f_{X(s, n, m, k) \mid X(r, n, m, k)}(y \mid x) \\
& =\frac{C_{s-1}}{(s-r-1) ! C_{r-1}} \\
& \quad \times \frac{\left[h_{m}(F(y))-h_{m}(F(x))\right]^{s-r-1}[\bar{F}(y)]^{\gamma_{s}-1}}{[\bar{F}(x)]^{\gamma_{r+1}}} f(y), x<y .
\end{aligned}
$$

Recurrence relations are interesting in their own right. They are useful in reducing the number of operations necessary to obtain a general form for the function under consideration. Furthermore, they are used in characterizing distributions, which in important area, permitting the identification of population distribution from the properties of the sample. Cramer and Kamps [5] derived relations for expectations of functions of generalized order statistics within a class of distributions including a variety of identities for single and product moments of ordinary order statistics and record values as particular cases. Various developments on gos and related topics have been studied by Kamps and Gather [6], Keseling [9], Cramer and Kamps [5], Pawlas and Szynal [16], Ahmad and Fawzy [1], Ahmad [2] and Kumar [13, 14, 15], among others. Khan and Kumar [10, 11, 12] have established recurrence relations for moments of lower generalized order statistics from exponentiated Pareto, gamma and generalized exponential distributions. Kamps [8] investigated the importance of recurrence relations for moments of order statistics in characterization.

The aim of the present study is to give exact expression and some recurrence relations for quotient moments of gos from generalized Pareto distribution. In Section 2, we give exact expression and recurrence relations for quotient moments of generalized Pareto distribution. Then we show that results for order statistics and record values are deduced as special cases. In Section 3, we give exact expression and recurrence relations for conditional quotient moments of generalized Pareto distribution and we show that results for order statistics and record values are deduced. In the last section of the paper we prove a characterization 
result on this distribution based on recurrence relation for conditional quotient moment of the gos.

A random variable $X$ is said to have generalized Pareto distribution if its $p d f$ is of the form

$$
f(x)=\frac{\alpha}{(\beta x+\alpha)^{2}}\left(\frac{\alpha}{\beta x+\alpha}\right)^{(1 / \beta)-1}, x>0, \alpha, \beta>0
$$

and the corresponding survival function is

$$
\bar{F}(x)=\left(\frac{\alpha}{\beta x+\alpha}\right)^{1 / \beta}, x>0, \alpha, \beta>0 .
$$

Here $\beta$ is the shape parameter. For $\beta>0$, the generalized Pareto distribution is known as Pareto type II or Lomax distribution . For $\beta=-1$, generalized Pareto distribution reduces uniform distribution on $(0, \alpha)$. As $\beta \rightarrow 0$, generalized Pareto distribution tends to exponential distribution with scale parameter $\alpha$. The generalized Pareto distribution is extensively used in the analysis of extreme values as well as in reliability studies when robustness is required against heavier tailed or lighter tailed alternatives to an exponential distribution. It is well known that the generalized Pareto distribution for $\beta>0$, provides reasonably good fit to distributions of income and property values. For more details and some applications of this distribution one may refer to Pickands [17] and Arnold [3].

\section{Relations for quotient moments}

Note that for generalized Pareto distribution defined in (1.6),

$$
\bar{F}(x)=(\beta x+\alpha) f(x) .
$$

The relation in (2.1) will be used to derive some simple recurrence relations for quotient moment of gos from the generalized Pareto distribution. These recurrence relations will be enable one to obtain all the quotient moments in a simple recursive manner.

Theorem 2.1. For generalized Pareto distribution as given in (1.6) and for $1 \leq r \leq s-2, k=1,2, \ldots, i=0,1,2, \ldots$ and $j=1,2, \ldots$, if $m \neq-1$ 


$$
\begin{aligned}
E & {\left[\frac{X^{i}(r, n, m, k)}{X^{j+1}(s, n, m, k)}\right] } \\
= & \frac{C_{s-1}}{(r-1) !(s-r-1) !(m+1)^{s-2}}\left(\frac{\beta}{\alpha}\right)^{j-i+1} \sum_{p=0}^{\infty} \sum_{q=0}^{i} \sum_{u=0}^{r-1} \sum_{v=0}^{s-r-1}(-1)^{u+v+q} \\
& \times\left(\begin{array}{c}
r-1 \\
u
\end{array}\right)\left(\begin{array}{c}
s-r-1 \\
v
\end{array}\right)\left(\begin{array}{c}
i \\
q
\end{array}\right) \frac{(j+1)_{(p)}}{p !\left[\gamma_{s-v}+\beta(p+j+1)\right]} \\
& \times \frac{1}{\left[\gamma_{r-u}+\beta(p+q+j-i+1)\right]}
\end{aligned}
$$

and if $m=-1$

$$
\begin{aligned}
& E\left[\frac{X^{i}(r, n,-1, k)}{X^{j+1}(s, n,-1, k)}\right] \\
& =k^{s}\left(\frac{\beta}{\alpha}\right)^{j-i+1} \sum_{p=0}^{\infty} \sum_{q=0}^{i}(-1)^{q}\left(\begin{array}{c}
i \\
q
\end{array}\right) \\
& \quad \times \frac{(j+1)_{(p)}}{p ![k+\beta(p+j+1)]^{s-r}[k+\beta(p+q+j-i+1)]^{r}}
\end{aligned}
$$

where

$$
(j)_{p}=\left\{\begin{array}{l}
j(j+1) \ldots(j+p-1), p>0 \\
1, p=0
\end{array} .\right.
$$

Proof. When $m \neq-1$, from (1.3), we have

$$
\begin{aligned}
& E\left[\frac{X^{i}(r, n, m, k)}{X^{j+1}(s, n, m, k)}\right] \\
& =\frac{C_{s-1}}{(r-1) !(s-r-1) !} \int_{0}^{\infty} \int_{x}^{\infty} \frac{x^{i}}{y^{j+1}}[\bar{F}(x)]^{m} f(x) \\
& \quad \times g_{m}^{r-1}(F(x))\left[h_{m}(F(y))-h_{m}(F(x))\right]^{s-r-1}[\bar{F}(y)]^{\gamma_{s}-1} f(y) d y d x .
\end{aligned}
$$

On using binomial expansion, (2.4), can be obtained when $m \neq-1$ as 


$$
\begin{aligned}
& E\left[\frac{X^{i}(r, n, m, k)}{X^{j+1}(s, n, m, k)}\right] \\
& =\frac{C_{s-1}}{(r-1) !(s-r-1) !(m+1)^{s-2}} \sum_{u=0}^{r-1} \sum_{v=0}^{s-r-1}(-1)^{u+v}\left(\begin{array}{c}
r-1 \\
u
\end{array}\right) \\
& \quad \times\left(\begin{array}{c}
s-r-1 \\
v
\end{array}\right) \int_{0}^{\infty} x^{i}[\bar{F}(x)]^{(s-r+u-v)(m+1)-1} f(x) G(x) d x,
\end{aligned}
$$

where

$$
G(x)=\int_{x}^{\infty} y^{-(j+1)}[\bar{F}(y)]^{\gamma_{s-v}-1} f(y) d y .
$$

By setting $t=[\bar{F}(y)]^{\beta}$ in (2.6), we get

$$
\begin{aligned}
G(x) & =\frac{\beta^{j}}{\alpha^{j+1}} \int_{x}^{[\bar{F}(x)]^{\beta}}(1-t)^{-(j+1)} t^{\frac{\beta(j+1)+\gamma_{s-v}}{\beta}-1} d t \\
& =\frac{\beta^{j}}{\alpha^{j+1}} \sum_{p=0}^{\infty} \frac{(j+1)_{(p)}}{p !} \int_{x}^{[\bar{F}(x)]^{\beta}} t^{\frac{\beta(j+1+p)+\gamma_{s-v}}{\beta}-1} d t \\
& =\left(\frac{\beta}{\alpha}\right)^{j+1} \sum_{p=0}^{\infty} \frac{(j+1)_{(p)}}{p !} \frac{[\bar{F}(x)]^{\gamma_{s-v}+\beta(p+j+1)}}{\left[\gamma_{s-v}+\beta(p+j+1)\right]} .
\end{aligned}
$$

On substituting the above expression of $G(x)$ in $(2.5)$, we find that

$$
\begin{aligned}
& E\left[\frac{X^{i}(r, n, m, k)}{X^{j+1}(s, n, m, k)}\right] \\
& =\frac{C_{s-1}}{(r-1) !(s-r-1) !(m+1)^{s-2}}\left(\frac{\beta}{\alpha}\right)^{j+1} \sum_{p=0}^{\infty} \sum_{u=0}^{r-1} \sum_{v=0}^{s-r-1} \\
& \quad \times(-1)^{u+v}\left(\begin{array}{c}
r-1 \\
u
\end{array}\right)\left(\begin{array}{c}
s-r-1 \\
v
\end{array}\right) \frac{(j+1)_{(p)}}{p !\left[\gamma_{s-v}+\beta(p+j+1)\right]} \\
& \quad \times \int_{0}^{\infty} x^{i}[\bar{F}(x)]^{\gamma_{r-u}+\beta(p+j+1)-1} f(x) d x .
\end{aligned}
$$

Again by setting $z=[\bar{F}(x)]^{\beta}$ in (2.7) and simplifying the resulting equation, we get the result given in (2.2).

When $m=-1$, we have that 


$$
\begin{aligned}
& E\left[\frac{X^{i}(r, n,-1, k)}{X^{j+1}(s, n,-1, k)}\right] \\
& =\frac{k^{s}}{(r-1) !(s-r-1) !} \int_{0}^{\infty} x^{i}[-\ln \bar{F}(x)]^{r-1} \frac{f(x)}{\bar{F}(x)} I(x) d x,
\end{aligned}
$$

where

$$
I(x)=\int_{x}^{\infty} y^{-(j+1)}[\ln (\bar{F}(x))-\ln (\bar{F}(y))]^{s-r-1}[\bar{F}(y)]^{k-1} f(y) d y .
$$

Setting $w=\ln (\bar{F}(x))-\ln (\bar{F}(y))$, we find that

$$
I(x)=\left(\frac{\beta}{\alpha}\right)^{j+1} \sum_{p=0}^{\infty} \frac{(j+1)_{(p)}}{p !} \frac{[\bar{F}(x)]^{k+\beta(p+j+1)} \Gamma(s-r)}{[k+\beta(p+j+1)]^{s-r}} .
$$

On substituting the above expression of $I(x)$ in (2.8), we obtain

$$
\begin{aligned}
& E\left[\frac{X^{i}(r, n,-1, k)}{X^{j+1}(s, n,-1, k)}\right] \\
& =\frac{k^{s}}{(r-1) !}\left(\frac{\beta}{\alpha}\right)^{j+1} \sum_{p=0}^{\infty} \frac{(j+1)_{(p)}}{p ![k+\beta(p+j+1)]^{s-r}} \\
& \quad \times \int_{0}^{\infty} x^{i}[-\ln \bar{F}(x)]^{r-1}[\bar{F}(x)]^{k+\beta(p+j+1)-1} f(x) d x .
\end{aligned}
$$

Again by setting $z=-\ln (\bar{F}(x))$ in (2.9) and simplifying the resulting expression, we get the result given in (2.3).

\section{Special cases}

i) Putting $m=0, k=1$ in (2.2), the exact expression for the quotient moments of order statistics of the generalized Pareto distribution is obtained as

$$
\begin{aligned}
E\left[\frac{X_{r: n}^{i}}{X_{s: n}^{j+1}}\right]= & C_{r, s: n}\left(\frac{\beta}{\alpha}\right)^{j-i+1} \sum_{p=0}^{\infty} \sum_{q=0}^{i} \sum_{u=0}^{r-1} \sum_{v=0}^{s-r-1}(-1)^{u+v+q}\left(\begin{array}{c}
r-1 \\
u
\end{array}\right) \\
& \times\left(\begin{array}{c}
s-r-1 \\
v
\end{array}\right)\left(\begin{array}{c}
i \\
q
\end{array}\right) \frac{(j+1)_{(p)}}{p ![n-s+1+v+\beta(p+j+1)]} \\
& \times \frac{1}{[n-r+1+u+\beta(p+q+j-i+1)]},
\end{aligned}
$$

where

$$
C_{r, s: n}=\frac{n !}{(r-1) !(s-r-1) !(n-s) !} .
$$


ii) Putting $k=1$ in (2.3), we deduce the explicit expression for the quotient moments of upper record values for generalized Pareto distribution in the form

$$
\begin{aligned}
E\left[\frac{X_{U(r)}^{i}}{X_{U(s)}^{j+1}}\right]= & \left(\frac{\beta}{\alpha}\right)^{j-i+1} \sum_{p=0}^{\infty} \sum_{q=0}^{i}(-1)^{q}\left(\begin{array}{c}
i \\
q
\end{array}\right) \\
& \times \frac{(j+1)_{(p)}}{p ![\beta(p+j+1)]^{s-r}[\beta(p+q+j-i+1)]^{r}} .
\end{aligned}
$$

Making use of (2.1), we can derive recurrence relations for the quotient moments of gos from (1.4).

Theorem 2.2. For $1 \leq r \leq s-2, k \geq 1, i=0,1,2, \ldots$ and $j=$ $1,2, \ldots$,

$$
\begin{aligned}
& \left(1+\frac{\beta(j+1)}{\gamma_{s}}\right) E\left[\frac{X^{i}(r, n, m, k)}{X^{j+1}(s, n, m, k)}\right] \\
& =E\left[\frac{X^{i}(r, n, m, k)}{X^{j+1}(s-1, n, m, k)}\right]-\frac{\alpha(j+1)}{\gamma_{s}} E\left[\frac{X^{i}(r, n, m, k)}{X^{j+2}(s, n, m, k)}\right] .
\end{aligned}
$$

Proof. We have from (1.3)

$$
\begin{aligned}
& E\left[\frac{X^{i}(r, n, m, k)}{X^{j+1}(s, n, m, k)}\right] \\
& =\frac{C_{s-1}}{(r-1) !(s-r-1) !} \int_{0}^{\infty} x^{i}[\bar{F}(x)]^{m} f(x) g_{m}^{r-1}(F(x)) I(x) d x
\end{aligned}
$$

where

$$
I(x)=\int_{x}^{\infty} \frac{1}{y^{j+1}}\left[h_{m}(F(y))-h_{m}(F(x))\right]^{s-r-1}[\bar{F}(y)]^{\gamma_{s}-1} f(y) d y .
$$

Integrating $I(x)$ by parts treating $[\bar{F}(y)]^{\gamma_{s}-1} f(y)$ for integration and the rest of the integrand for differentiation, we get

$I(x)$

$$
\begin{aligned}
= & -\frac{(j+1)}{\gamma_{s}} \int_{x}^{\infty} \frac{1}{y^{j+2}}\left[h_{m}(F(y))-h_{m}(F(x))\right]^{s-r-1}[\bar{F}(y)]^{\gamma_{s}} d y \\
& +\frac{(s-r-1)}{\gamma_{s}} \int_{x}^{\infty} \frac{1}{y^{j+1}}\left[h_{m}(F(y))-h_{m}(F(x))\right]^{s-r-2}[\bar{F}(y)]^{\gamma_{s}+m} f(y) d y .
\end{aligned}
$$

Substituting the value of $I(x)$ in (2.11) and simplifying the resulting expression we get the result given in (2.10). 
Remark 2.3. Putting $m=0$ and $k=1$ in (2.10), we obtain a recurrence relation for quotient moment of order statistics as

$$
\left(1+\frac{\beta(j+1)}{(n-s+1)}\right) E\left(\frac{X_{r: n}^{i}}{X_{s: n}^{j+1}}\right)=E\left(\frac{X_{r: n}^{i}}{X_{s-1: n}^{j+1}}\right)-\frac{\alpha(j+1)}{(n-s+1)} E\left(\frac{X_{r: n}^{i}}{X_{s: n}^{j+2}}\right) .
$$

Remark 2.4. Setting $m=-1$ and $k \geq 1$ in Theorem 2.2, we get a recurrence relation for quotient moment of upper $k$ record as

$$
\left(1+\frac{\beta(j+1)}{k}\right) E\left(\frac{X_{U(r): k}^{i}}{X_{U(s): k}^{j+1}}\right)=E\left(\frac{X_{U(r): k}^{i}}{X_{U(s-1): k}^{j+1}}\right)-\frac{\alpha(j+1)}{k} E\left(\frac{X_{U(r): k}^{i}}{X_{U(s): k}^{j+2}}\right) .
$$

\section{Relations for quotient conditional expectation}

Theorem 3.1. For the distribution as given in (1.6) and for $1 \leq r$ $<s \leq n-2, j=1,2, \ldots$, and $k=1,2, \ldots$, if $m \neq-1$

$$
\begin{aligned}
& E\left[\frac{1}{X^{j}(s, n, m, k)} \mid X(r, n, m, k)=x\right] \\
& =\left(-\frac{\beta}{\alpha}\right)^{j} \sum_{p=0}^{\infty} \frac{(j)_{p}}{p !}\left(\frac{\beta x+\alpha}{\alpha}\right)^{p} \prod_{w=1}^{s-r}\left(\frac{\gamma_{r+w}}{\gamma_{r+w}-\beta p}\right),
\end{aligned}
$$

and if $m=-1$

$$
\begin{aligned}
& E\left[\frac{1}{X^{j}(s, n,-1, k)} \mid X(r, n,-1, k)=x\right] \\
& =\left(\frac{\beta}{\alpha}\right)^{j} \sum_{p=0}^{\infty} \frac{(j)_{p}}{p !}\left(\frac{k}{\beta(p+j)}\right)^{s-r}\left(\frac{\alpha}{\beta x+\alpha}\right)^{p+j} .
\end{aligned}
$$

Proof. When $m \neq-1$ from (1.4), we have

$$
\begin{aligned}
& E\left[\frac{1}{X^{j}(s, n, m, k)} \mid X(r, n, m, k)=x\right] \\
& =\frac{C_{s-1}}{(s-r-1) ! C_{r-1}(m+1)^{s-r-1}} \\
& \quad \times \int_{x}^{\infty} y^{-j}\left[1-\left(\frac{\bar{F}(y)}{\bar{F}(x)}\right)^{m+1}\right]^{s-r-1}\left(\frac{\bar{F}(y)}{\bar{F}(x)}\right)^{\gamma_{s}-1} \frac{f(y)}{\bar{F}(x)} d y .
\end{aligned}
$$

By setting $u=\frac{\bar{F}(y)}{\bar{F}(x)}=\left(\frac{\beta x+\alpha}{\beta y+\alpha}\right)^{\frac{1}{\beta}}$ from (1.6) in (3.3), we obtain 


$$
\begin{aligned}
& E\left[\frac{1}{X^{j}(s, n, m, k)} \mid X(r, n, m, k)=x\right] \\
& =\frac{C_{s-1}}{(s-r-1) ! C_{r-1}(m+1)^{s-r-1}} \\
& \quad \times \int_{0}^{1}\left[-\frac{\beta}{\alpha}\left\{1-\frac{(\beta x+\alpha) u^{-\beta}}{\alpha}\right\}\right]^{-j} u^{\gamma_{s}-1}\left(1-u^{m+1}\right)^{s-r-1} d u \\
& =\frac{C_{s-1}}{(s-r-1) ! C_{r-1}(m+1)^{s-r-1}}\left(-\frac{\beta}{\alpha}\right)^{j} \sum_{p=0}^{\infty} \frac{(j)_{(p)}}{p !}\left(\frac{\beta x+\alpha}{\alpha}\right)^{p} \\
& \quad \times \int_{0}^{1} u^{\gamma_{s}-\beta p-1}\left(1-u^{m+1}\right)^{s-r-1} d u .
\end{aligned}
$$

Again by setting $t=u^{m+1}$ in (3.4), we get

$$
\begin{aligned}
& E\left[\frac{1}{X^{j}(s, n, m, k)} \mid X(r, n, m, k)=x\right] \\
& =\frac{C_{s-1}}{(s-r-1) ! C_{r-1}(m+1)^{s-r-1}}\left(-\frac{\beta}{\alpha}\right)^{j} \\
& \times \sum_{p=0}^{\infty} \frac{(j)_{p}}{p !}\left(\frac{\beta x+\alpha}{\alpha}\right)^{p} \int_{0}^{1} t^{\frac{k-\beta p}{(m+1)}+n-s-1}(1-t)^{s-r-1} d t \\
& =\frac{C_{s-1}}{(s-r-1) ! C_{r-1}(m+1)^{s-r}}\left(-\frac{\beta}{\alpha}\right)^{j} \sum_{p=0}^{\infty} \frac{(j)_{(p)}}{p !}\left(\frac{\beta x+\alpha}{\alpha}\right)^{p} \\
& \times \frac{\Gamma\left(\frac{k-\beta p}{(m+1)}+n-s\right) \Gamma(s-r)}{\Gamma\left(\frac{k-\beta p}{(m+1)}+n-r\right)} \\
& =\frac{C_{s-1}}{(s-r-1) ! C_{r-1}(m+1)^{s-r}}\left(-\frac{\beta}{\alpha}\right)^{j} \sum_{p=0}^{\infty} \frac{(j)_{(p)}}{p !}\left(\frac{\beta x+\alpha}{\alpha}\right)^{p} \\
& \times \frac{(m+1)^{s-r} \Gamma(s-r)}{\prod_{w=1}^{s-r}[(k-\beta p)+(n-r-w)(m+1)]} \\
& =\frac{C_{s-1}}{C_{r-1}}\left(-\frac{\beta}{\alpha}\right)^{j} \sum_{p=0}^{\infty} \frac{(j)_{(p)}}{p !}\left(\frac{\beta x+\alpha}{\alpha}\right)^{p} \frac{1}{\prod_{w=1}^{s-r}\left(\gamma_{r+w}-\beta p\right)},
\end{aligned}
$$

and hence the result given in (3.1).

When $m=-1$, we have that 


$$
\begin{aligned}
& E\left[\frac{1}{X^{j}(s, n,-1, k)} \mid X(r, n,-1, k)=x\right] \\
& =\frac{k^{s-r}}{(s-r-1) ![\bar{F}(x)]^{k}} \\
& \quad \times \int_{x}^{\infty} y^{-j}[\ln (\bar{F}(x))-\ln (\bar{F}(y))]^{s-r-1}[\bar{F}(y)]^{k-1} f(y) d y .
\end{aligned}
$$

Setting $w=\ln (\bar{F}(x))-\ln (\bar{F}(y))$ in (3.5) and integrating the resulting expression we get the result given in (3.2).

\section{Special cases}

i) Putting $m=0, k=1$ in (3.1), the exact expression for the conditional quotient moments of order statistics of the generalized Pareto distribution is obtained as

$$
\begin{aligned}
& E\left(\frac{1}{X_{s: n}^{j}} \mid X_{r: n}=x\right) \\
& =\left(-\frac{\beta}{\alpha}\right)^{j} \sum_{p=0}^{\infty} \frac{(j)_{(p)}}{p !}\left(\frac{\beta x+\alpha}{\alpha}\right)^{p} \times \prod_{w=1}^{s-r}\left(\frac{n-r-w+1}{n-r-w+1-\beta p}\right) .
\end{aligned}
$$

ii) Putting $k=1$ in (3.2), we deduce the explicit expression for the conditional quotient moments of upper record values for generalized Pareto distribution in the form

$$
E\left(\frac{1}{X_{U(s)}^{j}} \mid X_{U(r)}=x\right)=\left(\frac{\beta}{\alpha}\right)^{j} \sum_{p=0}^{\infty} \frac{(j)_{(p)}}{p !}\left(\frac{1}{\beta(p+j)}\right)^{s-r}\left(\frac{\alpha}{\beta x+\alpha}\right)^{p+j} .
$$

Making use of (2.1), we can derive recurrence relations for the conditional quotient moments of gos.

Theorem 3.2. For $1 \leq r \leq s-2, k \geq 1, i=0,1,2, \ldots$ and $j=1,2, \ldots$

$$
\begin{aligned}
& \left(1+\frac{\beta(j+1)}{\gamma_{s}}\right) E\left[\frac{X^{i}(r, n, m, k)}{X^{j+1}(s, n, m, k)} \mid X(r, n, m, k)=x\right] \\
& =E\left[\frac{X^{i}(r, n, m, k)}{X^{j+1}(s-1, n, m, k)} \mid X(r, n, m, k)=x\right] \\
& \quad-\frac{\alpha(j+1)}{\gamma_{s}} E\left[\frac{X^{i}(r, n, m, k)}{X^{j+2}(s, n, m, k)} \mid X(r, n, m, k)=x\right] .
\end{aligned}
$$


Proof. From (1.4), we have

$$
E\left[\frac{X^{i}(r, n, m, k)}{X^{j+1}(s, n, m, k)} \mid X(r, n, m, k)=x\right]=\frac{x^{i} C_{s-1} I(x)}{(s-r-1) ! C_{r-1}[\bar{F}(x)]^{\gamma_{r+1}}},
$$

where $I(x)$ is defined in (2.12). Substituting the value of $I(x)$ from (2.13) in (3.7) and simplifying the resulting expression we get the result given in (3.6).

Remark 3.3. Putting $m=0, k=1$ in (3.6), we obtain a recurrence relation for conditional quotient moment of order statistics as

$$
\begin{aligned}
& \left(1+\frac{\beta(j+1)}{n-s+1}\right) E\left(\frac{X_{r: n}^{i}}{X_{s: n}^{j+1}} \mid X_{r: n}=x\right) \\
& =E\left(\frac{X_{r: n}^{i}}{X_{s-1: n}^{j+1}} \mid X_{r: n}=x\right)-\frac{\alpha(j+1)}{(n-s+1)} E\left(\frac{X_{r: n}^{i}}{X_{s: n}^{j+2}} \mid X_{r: n}=x\right) .
\end{aligned}
$$

Remark 3.4. Setting $m=-1$ and $k \geq 1$ in Theorem 3.2, we get a recurrence relation for quotient moment of upper $k$ records as

$$
\begin{aligned}
& \left(1+\frac{\beta(j+1)}{k}\right) E\left(\frac{X_{U(r): k}^{i}}{X_{U(s): k}^{j+1}} \mid X_{U(r)}=x\right) \\
& =E\left(\frac{X_{U(r): k}^{i}}{X_{U(s-1): k}^{j+1}} \mid X_{U(r)}=x\right)-\frac{\alpha(j+1)}{k} E\left(\frac{X_{U(r): k}^{i}}{X_{U(s): k}^{j+2}} \mid X_{U(r)}=x\right) .
\end{aligned}
$$

\section{Characterization}

Theorem 4.1. Let $X$ be a non-negative random variable having an absolutely continuous distribution function $F(x)$ with $F(0)=0$ and $0<F(x)<1$ for all $x>0$, then

$$
\begin{aligned}
& E\left[\frac{1}{X^{j}(s, n, m, k)} \mid X(r, n, m, k)=x\right] \\
& =\left(-\frac{\beta}{\alpha}\right)^{j} \sum_{p=0}^{\infty} \frac{(j)_{(p)}}{p !}\left(\frac{\beta x+\alpha}{\alpha}\right)^{p} \prod_{w=1}^{s-r}\left(\frac{\gamma_{r+w}}{\gamma_{r+w}-\beta p}\right),
\end{aligned}
$$

if and only if

$$
\bar{F}(x)=\left(\frac{\alpha}{\beta x+\alpha}\right)^{1 / \beta}, x>0, \alpha, \beta>0 .
$$


Proof. The necessary part follows immediately from (3.1). On the other hand if (4.1) is satisfied, then on using equation (1.4), we have

$$
\begin{aligned}
\frac{C_{s-1}}{(s-r-1) ! C_{r-1}(m+1)^{s-r-1}} \int_{x}^{\infty} & y^{-j}\left[(\bar{F}(x))^{m+1}-(\bar{F}(y))^{m+1}\right]^{s-r-1} \\
& \times[\bar{F}(y)]^{\gamma_{s}-1} f(y) d y=[\bar{F}(x)]^{\gamma_{r+1}} H_{r}(x),
\end{aligned}
$$

where

$$
H_{r}(x)=\left(-\frac{\beta}{\alpha}\right)^{j} \sum_{p=0}^{\infty} \frac{(j)_{(p)}}{p !}\left(\frac{\beta x+\alpha}{\alpha}\right)^{p} \prod_{w=1}^{s-r}\left(\frac{\gamma_{r+w}}{\gamma_{r+w}-\beta p}\right) .
$$

Differentiating (4.2) both the sides with respect to $x$, and rearranging the terms, we get

$$
\begin{gathered}
-\frac{C_{s-1}[\bar{F}(x)]^{m} f(x)}{(s-r-2) ! C_{r-1}(m+1)^{s-r-2}} \int_{x}^{\infty} y^{-j}\left[(\bar{F}(x))^{m+1}-(\bar{F}(y))^{m+1}\right]^{s-r-2} \\
\quad \times[\bar{F}(y)]^{\gamma_{s}-1} f(y) d y=H_{r}^{\prime}(x)[\bar{F}(x)]^{\gamma_{r+1}}-\gamma_{r+1} H_{r}(x)[\bar{F}(x)]^{\gamma_{r+1}-1} f(x)
\end{gathered}
$$

or

$$
\begin{aligned}
& \gamma_{r+1} H_{r+1}(x)[\bar{F}(x)]^{\gamma_{r+2}+m} f(x) \\
& =H_{r}^{\prime}(x)[\bar{F}(x)]^{\gamma_{r+1}}-\gamma_{r+1} H_{r}(x)[\bar{F}(x)]^{\gamma_{r+1}-1} f(x) .
\end{aligned}
$$

Therefore

$$
\frac{f(x)}{\bar{F}(x)}=-\frac{H_{r}^{\prime}(x)}{\gamma_{r+1}\left[H_{r+1}(x)-H_{r}(x)\right]}=\frac{1}{\beta x+\alpha},
$$

where

$$
H_{r}^{\prime}(x)=\left(-\frac{\beta}{\alpha}\right)^{j} \sum_{p=0}^{\infty} \frac{\beta p(j)_{(p)}}{p ! \beta}\left(\frac{\beta x+\alpha}{\alpha}\right)^{p-1} \prod_{w=1}^{s-r}\left(\frac{\gamma_{r+w}}{\gamma_{r+w}-\beta p}\right)
$$

and

$$
H_{r+1}(x)=\left(-\frac{\beta}{\alpha}\right)^{j} \sum_{p=0}^{\infty} \frac{(j)_{(p)}}{p !}\left(\frac{\beta x+\alpha}{\alpha}\right)^{p} \prod_{w=1}^{s-(r+1)}\left(\frac{\gamma_{r+1+w}}{\gamma_{r+1+w}-\beta p}\right),
$$

which proves that

$$
\bar{F}(x)=\left(\frac{\alpha}{\beta x+\alpha}\right)^{1 / \beta}, x>0, \alpha, \beta>0 .
$$




\section{Acknowledgement.}

The author is grateful to acknowledge with thanks to referees for carefully reading the paper and for helpful suggestions which greatly improved the paper.

\section{References}

[1] A. A. Ahmad and A. M. Fawzy, Recurrence relations for single moments of generalized order statistics from doubly truncated distributions, J. Statist. Plann. Inference 117 (2003), 241-249.

[2] A. A. Ahmad, Recurrence relations for single and product moments of generalized order statistics from doubly truncated Burr type XII distribution, J. Egypt. Math. Soc. 15 (2007), 117-128.

[3] B. C. Arnold, The Pareto distribution, International Co-operative Publishing Houshing, Fairland, MD., 1983.

[4] E Crammer, Contributions to generalized order statistics, Ph. D. Thesis. University of Oldenburg., 2002.

[5] E. Camer and U. Kamps, Relations for expectations of functions of generalized order statistics, J. Statist. Plann. Inference 89 (2000), 79-89.

[6] U. Kamps and U. Gather, Characteristic property of generalized order statistics for exponential distribution, Appl. Math. 24 (1997), 383-391.

[7] U. Kamps, A concept of Generalized Order Statistics, B.G. Teubner Stuttgart., 1995.

[8] U. Kamps, Characterizations of distributions by recurrence relations and identities for moments of order statistics. In: Balakrishnan, N. And C.Rao, C.R. eds., Hand book of statistics, 16, Order Statistics, Theory and Methods, Amsterdam: North Holand, 16 (1998), 291-231.

[9] C. Keseling, Conditional distributions of generalized order statistics and some characterizations, Metrika 49 (1999), 27-40.

[10] R. U. Khan amd D. Kumar, On moments of lower generalized order statistics from exponentiated Pareto distribution and its characterization, Appl. Math. Sci. 4 (2010), 2711-2722.

[11] R. U. Khan amd D. Kumar, Lower generalized order statistics from exponentiated gamma distribution and its characterization, ProbStats Forum 4 (2011), 25-38.

[12] R. U. Khan amd D. Kumar, Expectation identities of lower generalized order statistics from generalized exponential distribution and a characterization, Math. Methods Statist. 20 (2011), 150-157.

[13] D. Kumar, Recurrence relations for single and product moments of generalized order statistics from $p$-th order exponential distribution and its characterization, Journal of Statistical Research of Iran 7 (2010), 101-112.

[14] D. Kumar, Generalized order statistics from Kumaraswamy distribution and its characterization, Tamsui Oxford Journal of Mathematical Sciences 27 (2011), 463-476. 
[15] D. Kumar, Relations of generalized order statistics from Erlang-Truncated exponential distribution, Pacific Journal of Applied Statistics 6 (2014), 55-77.

[16] P. Pawlas and D. Szynal, Recurrence relations for single and product moments of generalized order statistics from Pareto, generalized Pareto, and Burr distributions, Comm. Statist. Theory Methods 30 (2001), 739-746.

[17] J. Pickands, Staistical inference using extreme order statistics Ann. Statist 3 (1975), 119-131.

$*$

Department of Statistics, Amity Institute of Applied Sciences

Amity University

Noida-201 303, India

E-mail: devendrastats@gmail.com 